\title{
Tabu Search-Based Method for Bézier Curve Parameterization
}

\author{
Akemi Gálvez $^{1}$, Andrés Iglesias ${ }^{1,2, \dagger}$, Luis Cabellos ${ }^{3}$ \\ ${ }^{1}$ Department of Applied Mathematics \& Computational Sciences \\ E.T.S.I. Caminos, Canales y Puertos \\ University of Cantabria, Avda. de los Castros, s/n \\ E-39005, Santander, SPAIN \\ ${ }^{2}$ Department of Information Science, Faculty of Sciences \\ Toho University, 2-2-1 Miyama \\ 274-8510, Funabashi, Japan \\ ${ }^{3}$ IFCA-Instituto de Física de Cantabria \\ Avda. de los Castros, $s / n$ \\ E-39005, Santander, Spain \\ ${ }^{\dagger}$ Corresponding author: iglesias@unican.es \\ Web page: http://personales.unican.es/iglesias
}

\begin{abstract}
A very important issue in many applied fields is to construct the fitting curve that approximates a given set of data points optimally in the sense of least-squares. This problem arises in a number of areas, such as computer-aided design \& manufacturing (CAD/CAM), virtual reality, medical imaging, computer graphics, computer animation, and many others. This is also a hard problem, because it is highly nonlinear, over-determined and typically involves a large number of unknown variables. A critical step in this process is to obtain a suitable parameterization of the data points. In this context, this paper introduces a new method to obtain an optimal solution for the parameterization problem of the least-squares fitting Bézier curve. Our method is based on a local search metaheuristic approach for optimization problems called tabu search. The method is applied to some simple yet illustrative examples for the cases of $2 D$ and $3 D$ curves. The proposed method is simple to understand, easy to implement and can be applied to any kind of smooth data points. Our experimental results show that the presented method performs very well, being able to fit the data points with a high degree of accuracy.
\end{abstract}

\section{Introduction}

Fitting curves to data points is a very important issue in many applied fields such as computeraided design \& manufacturing (CAD/CAM), virtual reality, medical imaging, computer animation, and many others. Data can be either generated synthetically by direct application of a myriad of CAD/CAM computer programs or acquired from real measurements of an existing geometric entity, as it typically happens in the construction of car bodies, ship hulls, airplane fuselage and other free-form objects $[8,10,12,25,27,38,45]$. This problem also appears in the shoes industry, in archeology (reconstruction of archeological assets), in medicine (computer tomography) and in 
many other fields. In all those cases, it is desirable to obtain the fitting curve that approximates the set of data points optimally in the sense of least-squares.

Owing to their remarkable mathematical properties, polynomial curves are usually applied to tackle this issue. In particular, free-form parametric curves such as Bézier, B-spline and NURBS, are widely applied in many industrial settings due to their great flexibility and the fact that they can represent well any smooth shape with only a few parameters, thus leading to substantial savings in terms of computer memory and storage capacity. Best approximation methods make commonly use of least-squares techniques [11, 32, 33, 46]. In this paper we focus particularly in the case of Bézier curves, where the goal is to obtain the control points of the optimal fitting curve in the least-squares sense. This problem is far from being trivial; since the curve is parametric, we are confronted with the problem of obtaining a suitable parameterization of the data points [7, 13]. In fact, the selection of an appropriate parameterization is essential for a good fitting. Unfortunately, it is also a very hard problem. It is well-known that it leads to a difficult over-determined nonlinear optimization problem. It is also multivariate; in fact, it typically involves a large number of unknown variables for a large number of data points, a case that happens very often in real-world examples.

The problem of Bézier curve parameterization has been the subject of research for many years $[4,6,37]$. The usual goal is to obtain a parameterization as similar as possible to to the arc-length parameterization. The ultimate reason for this is that a constant step on the parametric domain automatically translates into a constant distance along an arc-length parameterized curve. In other words, for constant parameter intervals, the curve exhibits a point spacing that is as uniform as possible. Therefore, this parameterization is very convenient for curve and surface interrogation issues such as measuring distances on a surface. Further, since some industrial operations require an uniform parameterization, this property has several practical applications. For example, in computer controlled milling operations, the curve path followed by the milling machine must be parameterized such that the cutter neither speeds up nor slows down along the path [36]. This property is only guaranteed when the curve is parameterized with the arc-length parameterization. Consequently, this has been the most classical and most preferred choice for curve parameterization.

Some recent papers have shown that the application of Artificial Intelligence (AI) techniques can achieve remarkable results regarding many parameterization problems [2, 35, 42]. Consequently, a number of different methods, including metaheuristics such as Particle Swarm Optimization (PSO) [1, 3, 16, 34, 41], Genetic Algorithms (GA) [19, 21, 23, 30], Harmony Search (HS) [39, 47], Ant Colony Optimization (ACO) [43], Simulated Annealing (SA) [5], Bee Colony Optimization (BCO) [29], Clonal Selection Algorithm (CSA) [20], and many others. Some of these methods have been applied to the parameterization problem [8, 22, 24, 25, 27, 31]. Most of these methods rely on some kind of neural networks, such as standard neural networks [22] and Kohonen's SOM (Self-Organizing Maps) nets [24]. The generalization to functional networks is also analyzed in $[8,25,26,27,28]$. A previous paper in [9] describes the application of genetic algorithms and functional networks yielding pretty good results. Other approaches are based on the application of metaheuristic techniques, which have been intensively applied to solve difficult optimization problems that cannot be tackled through traditional optimization algorithms. Recent schemes in this area are described in [10,11,13] for particle swarm optimization (PSO), [12, 40, 48] for genetic algorithms (GA), [14, 44] for artificial immune systems, [49] for estimation of distribution algorithms, and [15] for hybrid GA-PSO techniques. 


\subsection{Aims and structure of the paper}

This paper introduces a new method to obtain an optimal solution for the parameterization problem of the least-squares fitting Bézier curve. Our method is based on a local search metaheuristic approach for optimization problems called tabu search. This metaheuristic approach is based on two driving ideas: the first one is the use of memory structures to store previously visited solutions which are not allowed to be re-visited again in order to prevent from stagnation and cycling. As it will be described later on, these memory structures storing non allowed moves are called tabu lists and play a central role in the method. The second main idea is that not only potential solutions but also attributes can be effectively used to drive the search for the optima in the problem space. When properly applied, the use of these attributes (or rules) provides a much improved procedure to manipulate the potential solutions, leading to a general improvement of the searching.

The structure of this paper is as follows: the principles and fundamentals of the tabu search, the metaheuristic used in this paper, are briefly explained in Section 2. Then, Section 3 describes the proposed method in detail. As the reader will see, the method is simple to understand, easy to implement and can be applied to any kind of smooth data points. To check the performance of our approach, it has been applied to four simple yet illustrative examples for the cases of 2D and 3D curves, as described in Section 4. Our experimental results show that the presented method performs very well, being able to yield the best approximating curve with a high degree of accuracy. The paper closes with the main conclusions of this contribution and our plans for future work in the field.

\section{Tabu Search}

Tabu Search is a metaheuristic technique originally developed by Fred W. Glover in 1986 to allow local search methods to overcome local optima [17]. The method is intended for discrete spaces although it can be adapted to continuous problems, as we will show later on. Typically, tabu search uses a local or neighborhood search procedure to iteratively move from one potential solution, $S$, (usually chosen randomly at the initial stage) to an improved solution, $S^{*}$, in the neighborhood of $S$, denoted by $N(S)$.

The basic principle of tabu search is to pursue local search whenever a local optimum is reached by allowing non-improving moves. In order to prevent the algorithm to reach into previously visited solutions (thus falling into a cycle), the method make use of memory structures, called tabu lists, whose aim is to record the recent history of the search. Basically, a tabu list is a set of banned solutions used to filter the solutions that will be admitted. In its simplest form, a tabu list is a shortterm set of the solutions that have been visited in the recent past (by short-term we mean a lifespan of the last $n$ iterations of the process, where $n$ is the number of previous solutions to be stored, usually called the tabu tenure; see [18] for details).

The search process can also be enriched with additional rules to drive the search towards promising areas of the search space as well as to promote diversity, playing the role of intermediate and long-term memories, respectively. From this point of view, tabu search can be seen as a clever combination of local search procedures with temporal memory structures. Furthermore, this memory is both explicit and attributive, meaning that not only explicit solutions but also rules and attributes can be included into the tabu lists. Indeed, in order to improve the effectiveness of tabu search, the tabu lists can contain attributes rather than (or, in addition to) solutions. However, this variant might introduce a new problem: tabu lists based on attributes may prohibit attractive moves, even when 
there is no danger of cycling, or they may lead to an overall stagnation of the searching process. Therefore, we need a procedure to revoke (cancel) tabus by allowing a move, even if it is tabu, when it results in a solution whose objective value is better than that of the current best-known solution according to a given measure, called fitness. This process is driven by the aspiration criteria, where each rule to overcome tabus behaves, in practice, as an aspiration criterion.

In general, for any specific target problem, there are many more possible (and even, attractive) neighborhood structures than search space definitions. This follows from the fact that there may be several plausible neighborhood structures for a given definition of the search space. In fact, once long-term memory is embedded into the model, tabu search may be viewed as a dynamic neighborhood method. This means that the neighborhood of any given solution is not a static set, but rather a set that can change according to the history of the search.

Once all tabu rules, lists and attributes are determined, the tabu search algorithm is executed until a termination criterion is satisfied. The most commonly used stopping criteria in tabu search are: (1) after a fixed number of iterations (or a fixed amount of CPU time); (2) after some number of iterations without any improvement in the objective function value (the criterion used in most implementations); or, (3) when the objective reaches a pre-specified threshold value, which is problem-dependent and usually specified by the user.

\section{Our method}

We assume that the reader is familiar with the main concepts of free-form parametric curves [7]. A free-form parametric Bézier curve $\mathbf{C}(t)$ of degree $n$ is defined as:

$$
\mathbf{C}(t)=\sum_{j=0}^{n} \mathbf{P}_{j} B_{j}^{n}(t)
$$

where $\mathbf{P}_{j}$ are vector coefficients (usually referred to as the control points), $B_{j}^{n}(t)$ are the Bernstein polynomials of index $j$ and degree $n$, given by:

$$
B_{j}^{n}(t)=\left(\begin{array}{c}
n \\
j
\end{array}\right) t^{j}(1-t)^{n-j}
$$

and $t$ is the curve parameter, defined on a finite interval $[0,1]$. Note that in this paper vectors are denoted in bold. By convention, $0 !=1$.

Let us suppose now that we are given a set of data points $\left\{\mathbf{Q}_{i}\right\}_{i=1, \ldots, m}$ in $\mathbb{R}^{d}$ (usually $d=2$ or $d=3$ ). Our goal is to obtain the free-form parametric Bézier curve $\mathbf{C}(t)$ that fits the data points better in the discrete least-squares sense. To do so, we have to compute the control points $\mathbf{P}_{j}$ $(j=0, \ldots, n)$ of the approximating curve $\mathbf{C}(t)$ by minimizing the least-squares error, $E$, defined as the sum of squares of the residuals:

$$
E=\sum_{i=1}^{m}\left(\mathbf{Q}_{i}-\sum_{j=0}^{n} \mathbf{P}_{j} B_{j}^{n}\left(t_{i}\right)\right)^{2}
$$

where we need a parameter value $t_{i}$ to be associated with each data point $\mathbf{Q}_{i}, i=1, \ldots, m$. Considering the column vectors $\mathbf{B}_{j}=\left(B_{j}^{n}\left(t_{1}\right), \ldots, B_{j}^{n}\left(t_{m}\right)\right)^{T}, j=0, \ldots, n$, where $(.)^{T}$ means transposition, and $\overline{\mathbf{Q}}=\left(\mathbf{Q}_{1}, \ldots, \mathbf{Q}_{m}\right)$, Eq. (2) becomes the following system of equations (called the normal equation): 


$$
\left(\begin{array}{ccc}
\mathbf{B}_{0}^{T} \cdot \mathbf{B}_{0} & \ldots & \mathbf{B}_{n}^{T} \cdot \mathbf{B}_{0} \\
\vdots & \vdots & \vdots \\
\mathbf{B}_{0}^{T} \cdot \mathbf{B}_{n} & \ldots & \mathbf{B}_{n}^{T} \cdot \mathbf{B}_{n}
\end{array}\right)\left(\begin{array}{c}
\mathbf{P}_{0} \\
\vdots \\
\mathbf{P}_{n}
\end{array}\right)=\left(\begin{array}{c}
\overline{\mathbf{Q}} \cdot \mathbf{B}_{0} \\
\vdots \\
\overline{\mathbf{Q}} \cdot \mathbf{B}_{n}
\end{array}\right)
$$

which can be compacted as:

$$
\mathbf{M} . \mathbf{P}=\mathbf{R}
$$

with $\mathbf{M}=\left[\sum_{j=1}^{m} B_{l}\left(t_{j}\right) B_{i}\left(t_{j}\right)\right]$ and $\mathbf{R}=\left[\sum_{j=1}^{m} \mathbf{Q}_{j} B_{l}\left(t_{j}\right)\right]$ for $i, l=0, \ldots, n$.

Due to the fact that the blending functions $B_{j}^{n}(t)$ are nonlinear in $t$, the least-squares minimization of the errors is a strongly nonlinear problem, with a high number of unknowns for large sets of data points, a case that happens very often in practice. On the other hand, if values are assigned to the $t_{i}$, the problem becomes a classical linear least-squares minimization, with the coefficients $\left\{\mathbf{Q}_{i}\right\}$ as unknowns. Therefore, it is clear that solving the parameterization problem is the key to obtain an optimal fitting Bézier curve of data.

Our strategy for solving such a problem consists of applying the taboo search algorithm to determine suitable parameter values for the least-squares minimization of functional $E$ according to (2). In this paper we consider a tabu list $L$ comprised of a fixed number $N$ of banned solutions, $N$ being a parameter of the method. The potential solutions are a collection of parametric vectors $\left\{\mathbf{T}_{j}\right\}_{j=1, \ldots, N}$, where each $\mathbf{T}_{j}=\left(t_{1}^{j}, t_{2}^{j}, \ldots, t_{m}^{j}\right) \in[0,1]^{m}$ and the $\left\{t_{i}^{j}\right\}_{i}$ are strictly increasing parameters. These parametric vectors are sorted according to the functional $E$ and the best $d$ solutions are selected for the tabu list $L$. Then, the best current solution undergoes further transformations, according to the following rule: a random integer $i \in\{1, \ldots, m\}$ is chosen; then, its $i$-th component is transformed as $t_{i}^{*}=\operatorname{rand}\left(t_{i-1}, t_{i+1}\right)$, where rand represents a uniform random variable in the given interval. This operation is repeated $p$ times, where $p$ is also a parameter of the method. The resulting $\mathbf{T}_{j}^{*}$ are then inserted into the list of potential solutions, which are subsequently ranked according to $E$, and the tabu list is updated accordingly. The process is performed iteratively for a given number of iterations, until the convergence of the minimization of the error is achieved.

\section{Experimental results}

This section discusses the performance of our tabu search-based method for Bézier curve parameterization through four simple yet illustrative examples, corresponding to the cases of bi- and three-dimensional curves. These examples have been carefully chosen to reflect the variety of situations our method can be applied to. Many other examples have also been tested with excellent results in all cases. They are not reported here because of limitations of space.

\subsection{Two-dimensional examples}

Figures 1 and 2 show two examples corresponding to two-dimensional curves: the epicycloid and the spiral hyperbolic tangent, respectively. As the reader can see, both have challenging features: the epicycloid curve has several non-differentiable turning points, while the spiral hyperbolic tangent curve exhibits several changes of concavity. In our figures, the original data points are displayed as red emptied circles whereas the reconstructed points appear as blue plus symbols. The number of data points for these two examples is $m=200$ and $m=300$, respectively. The following values for the parameters of the tabu search method have been used: $N=50, d=50$, and 


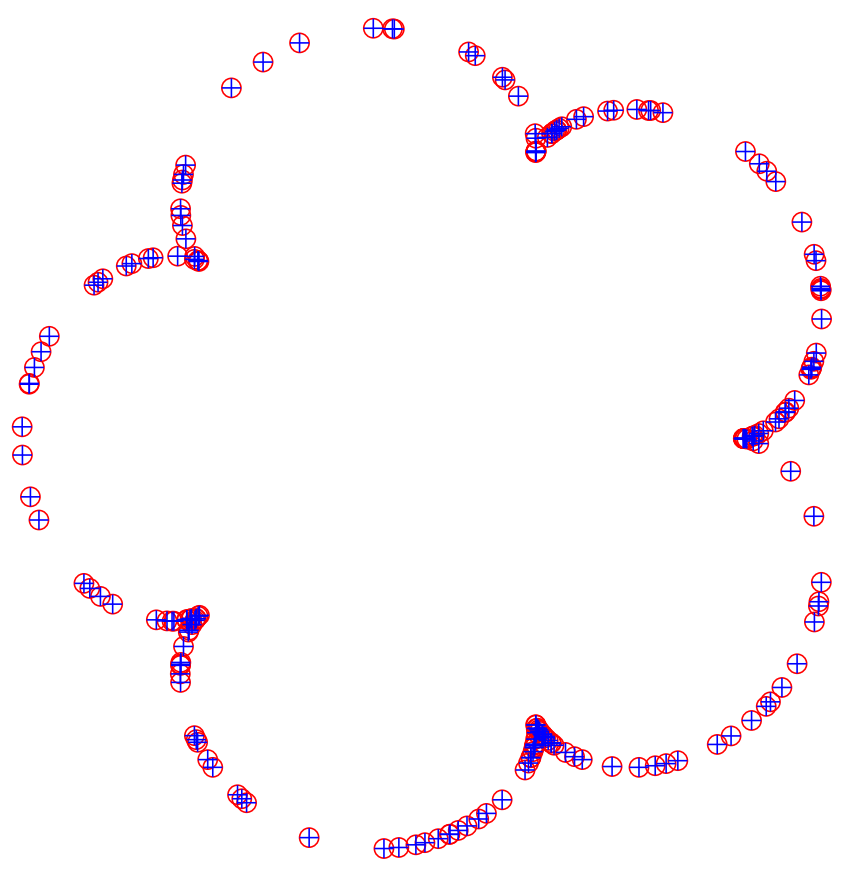

Figure 1. Applying the tabu search algorithm to a 2D Bézier curve parameterization: original (red emptied circles) and reconstructed (blue + symbols) data points for the epicycloid curve.

$p=30$. An initial population of 100 randomly chosen solutions is considered; then, the method is executed for 50 iterations. Note the excellent matching between the original and the reconstructed data points. In fact, we got a RMSE fitting error of $4.3 \times 10^{-6}$ for the epicycloid curve and $1.21 \times 10^{-5}$ for the spiral hyperbolic tangent, indicating that the reconstructed Bézier curve fits the data points with extremely high accuracy. We also computed the absolute mean value of the difference between the original and the reconstructed data points for each coordinate, and obtained excellent results: $\left(3.42 \times 10^{-6}, 4.78 \times 10^{-6}\right)$ (left) and $\left(0.98 \times 10^{-5}, 1.32 \times 10^{-6}\right)$.

\subsection{Three-dimensional examples}

Figures 3 and 4 show two examples corresponding to three-dimensional curves: the Viviani curve and the conic helix curve, respectively. We applied our method to these curves for the same parameter values with $m=100$ and $m=500$ data points, respectively. Once again, we obtained very good results, with a RMSE fitting error of $3.98 \times 10^{-5}$ for the Viviani curve and $4.76 \times 10^{-5}$ for the the conic helix curve, respectively. Note the excellent matching between the original and the fitted data points, which is clearly visible in all our figures.

\subsection{Computational issues}

All computations in this paper have been performed on a $2.9 \mathrm{GHz}$. Intel Core i7 processor with 8 GB. of RAM. The source code has been implemented by the authors in the native programming language of the popular scientific program Matlab, version 2010b. Regarding the computation times, all examples we tested can be obtained in less than a second (excluding rendering). Obviously, 


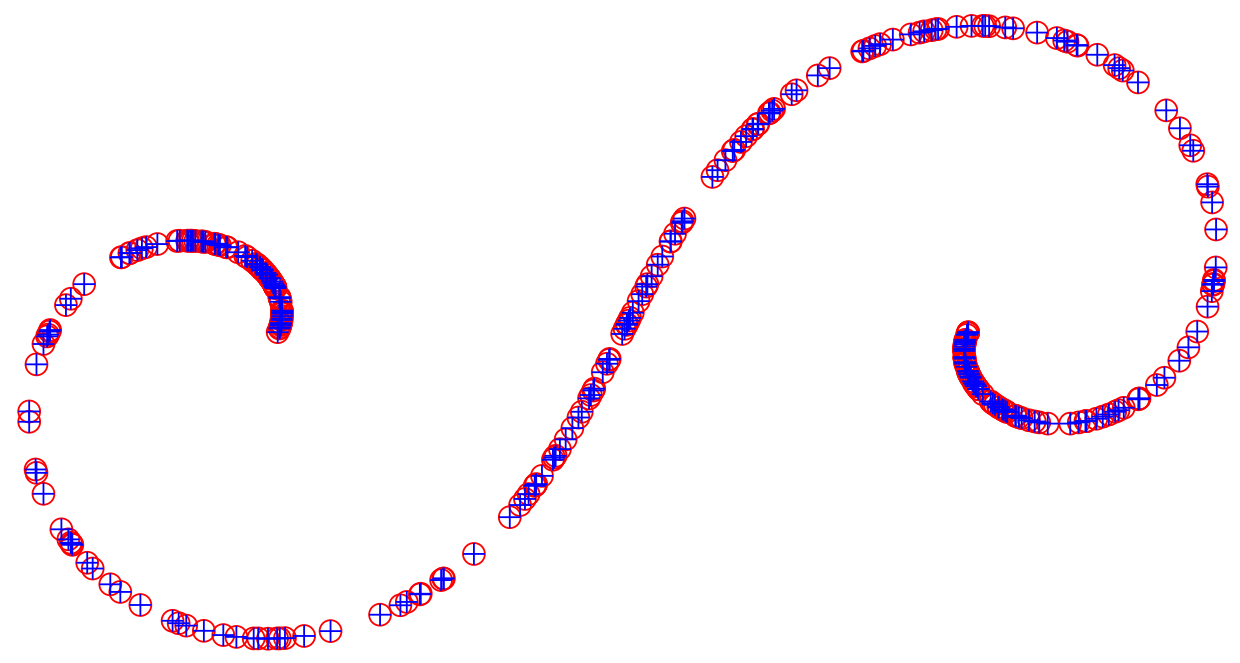

Figure 2. Applying the tabu search algorithm to a 2D Bézier curve parameterization: original (red emptied circles) and reconstructed (blue + symbols) data points for the spiral hyperbolic tangent curve.

the CPU time depends on several factors, such as the number of data points and the values of the different parameters of the method, making it hard to determine how long does it take for a given example to be reconstructed.

\section{Conclusions and future work}

This paper presents a tabu search-based method for Bézier curve parameterization. Given a set of data points, the method computes a suitable parameterization of data points in order to obtain the Bézier curve that fits the data points better in the least-squares sense. This problem is far from being trivial as soon as no parameterization of data points is assumed a priori. Furthermore, data points are subjected to noise in their parametric values (this fact is clearly noticeable from the simple visual inspection of the uneven distribution of data in all our figures), meaning that the uniform parameterization is by no means a feasible solution; instead, a proper non-trivial parameterization is actually required. The method is applied to some simple yet illustrative examples for the cases of 2D and 3D curves. Our experimental results show that the presented method performs very well, being able to fit the data points with a high degree of accuracy.

Future work includes the extension of this method to other families of parametric curves, such as the B-splines and NURBS, where the existence of additional parameters (such as knots and weights) can modify our procedure significantly. We are also working towards the applicability of this approach in several industrial problems.

\section{Acknowledgements}

This research has been financially supported by the Computer Science National Program of the Spanish Ministry of Economy and Competitiveness, Project Ref. \#TIN2012-30768, Toho Univer- 


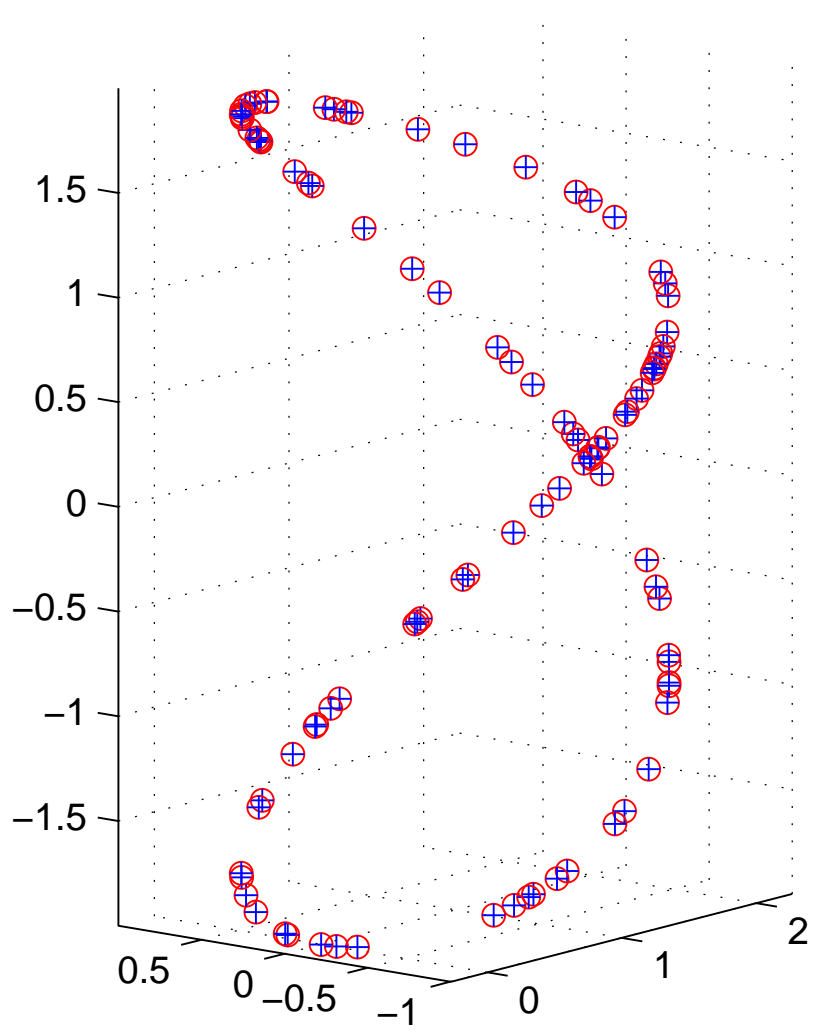

Figure 3. Applying the tabu search algorithm to a 3D Bézier curve parameterization: original (red emptied circles) and reconstructed (blue + symbols) data points for the Viviani curve.

sity, the University of Cantabria, and the Instituto de Física de Cantabria, a mixed research center of the University of Cantabria and CSIC-Consejo Superior de Investigaciones Científicas.

\section{References}

[1] Abdel-Kader, R.F.: An Improved Discrete PSO with GA Operators for Efficient QoS-Multicast Routing. International Journal of Hybrid Information Technology, 4 (2), (2011) 23-38.

[2] Baguda, Y.S., Fisal, N., Rashid, R.A., Yusof, S.K., Syed, S.H., Shuaibu, D.S.: Biologically-Inspired Optimal Video Streaming over Unpredictable Wireless Channel. International Journal of Future Generation Communication and Networking, 5(1), (2012) 15-28.

[3] El Bakrawy, L.M., Ghali, N.I., Kim, T.H., Hassanien, A.E.: A Block-wise-based Fragile Watermarking Hybrid Approach using Rough Sets and Exponential Particle Swarm Optimization. International Journal of Future Generation Communication and Networking, 4(4), (2011) 77-88.

[4] Barnhill, R.E.: Geometric Processing for Design and Manufacturing. SIAM, Philadelphia (1992)

[5] Benaddy, M., Wakrim, M.: Simulated Annealing Neural Network for Software Failure Prediction. International Journal of Software Engineering and Its Applications, 6(4), (2012) 35-46. 


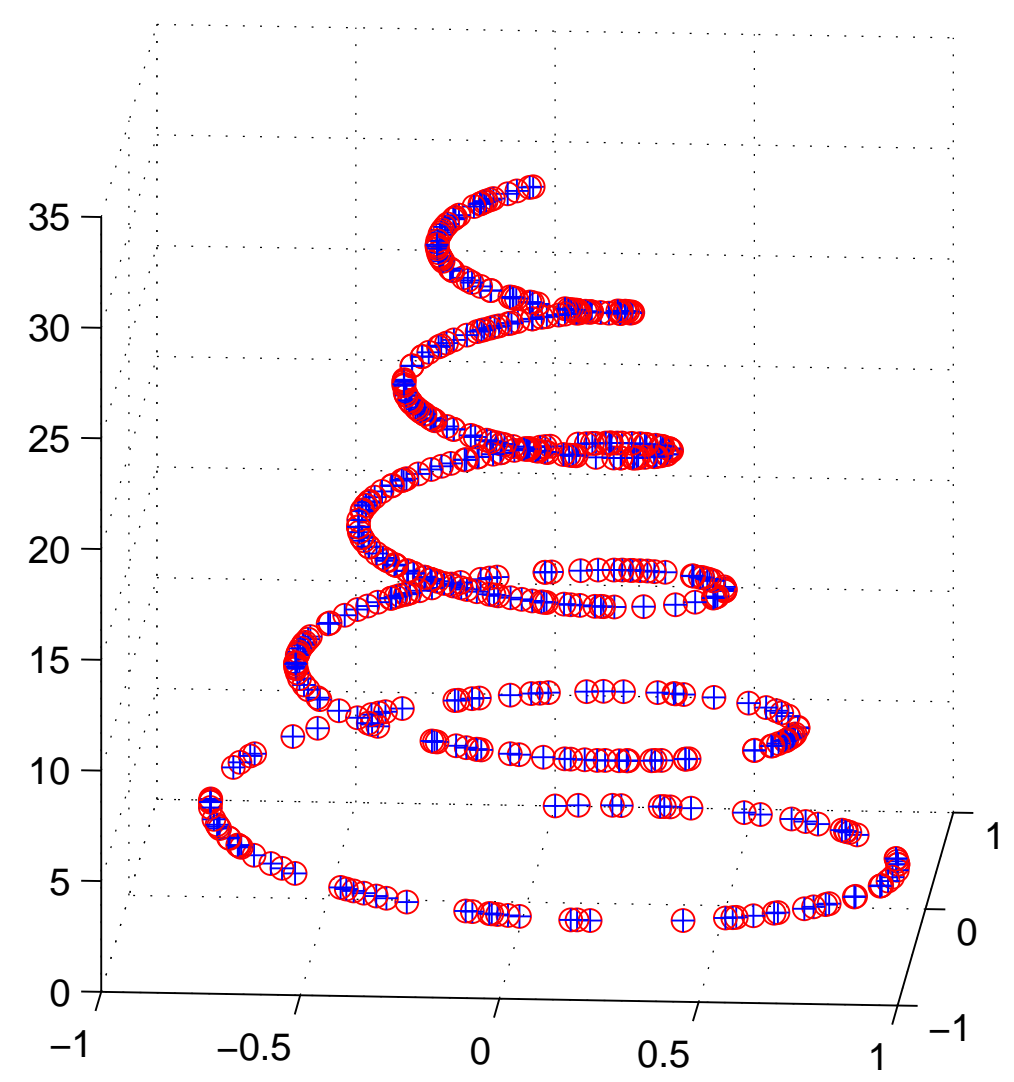

Figure 4. Applying the tabu search algorithm to a 3D Bézier curve parameterization: original (red emptied circles) and reconstructed (blue + symbols) data points for the conic helix curve.

[6] Dierckx, P.: Curve and Surface Fitting with Splines. Oxford University Press, Oxford (1993).

[7] Farin, G.: Curves and surfaces for CAGD (5th ed.). Morgan Kaufmann, San Francisco (2002).

[8] Echevarría, G., Iglesias, A., Gálvez, A.: Extending neural networks for B-spline surface reconstruction. Lectures Notes in Computer Science, 2330 (2002) 305-314.

[9] Gálvez, A., Iglesias, A., Cobo, A., Puig-Pey, J., Espinola, J.: Bézier curve and surface fitting of 3D point clouds through genetic algorithms, functional networks and least-squares approximation. Lectures Notes in Computer Science, 4706 (2007) 680-693.

[10] Gálvez, A., Cobo, A., Puig-Pey, J., Iglesias, A.: Particle swarm optimization for Bézier surface reconstruction. Lectures Notes in Computer Science, 5102 (2008) 116-125.

[11] Gálvez, A., Iglesias A.: Efficient particle swarm optimization approach for data fitting with free knot B-splines. Computer-Aided Design, 43(12) (2011) 1683-1692. 
[12] Gálvez, A., Iglesias A., Puig-Pey J.: Iterative two-step genetic-algorithm method for efficient polynomial B-spline surface reconstruction. Information Sciences, 182(1) (2012) 56-76.

[13] Gálvez A., Iglesias A.: Particle swarm optimization for non-uniform rational B-spline surface reconstruction from clouds of 3D data points. Information Sciences, 192(1) (2012) 174-192.

[14] Gálvez A., Iglesias A., Andreina, A.: Discrete Bézier Curve Fitting with Artificial Immune Systems. Studies in Computational Intelligence, 441 (2013) 59-75.

[15] Gálvez A., Iglesias A.: A New Iterative Mutually-Coupled Hybrid GA-PSO Approach for Curve Fitting in Manufacturing. Applied Soft Computing, 13(3) (2013) 1491-1504.

[16] Gao, X. Z., Wu, Y., Huang, X., Zenger, K.: A Novel Cultural Quantum-behaved Particle Swarm Optimization Algorithm. International Journal of Hybrid Information Technology, 5(2), (2012) 117 122.

[17] Glover, F.W., McMillan, C.: The general employee scheduling problem: an integration of MS and AI. Computers and Operations Research, 13(5) (1986) 563-573.

[18] Glover, F.W., Laguna, M.: Tabu Search. Kluwer Academic Publishers, Norwell, MA (1997).

[19] Giordano, J., Burtschell, Y.: Confrontation of Genetic Algorithm Optimization Process with a New Reference Case: Analytical Study with Experimental Validation of the Deflection of a Cantilever Beam. International Journal of Advanced Science and Technology, 49 (2012) 105-118.

[20] Gong, T., Li, T., Guo, C., Gong, X.: Novel Clonal Selection Algorithm Improving Selection Operator. International Journal of Multimedia and Ubiquitous Engineering, 7(2), (2012) 323-328.

[21] Goyal, S., Gupta, R.: Optimization of Fidelity with Adaptive Genetic Watermarking Algorithm using Tournament Selection. International Journal of Advanced Science and Technology, 30 (2011) 55-66.

[22] Gu, P., Yan, X.: Neural network approach to the reconstruction of free-form surfaces for reverse engineering. Computer-Aided Design 27(1) 59-64 (1995)

[23] Heidari, H., Chalechale, A.: Scheduling in Multiprocessor System Using Genetic Algorithm. International Journal of Advanced Science and Technology, 43 (2012) 81-94.

[24] Hoffmann M.: Numerical control of Kohonen neural network for scattered data approximation. $\mathrm{Nu}$ merical Algorithms, 39 (2005) 175-186.

[25] Iglesias, A., Gálvez, A.: A new artificial intelligence paradigm for computer aided geometric design. Lectures Notes in Artificial Intelligence, 1930 (2001) 200-213.

[26] Iglesias, A., Gálvez, A.:Applying functional networks to fit data points rom B-spline surfaces. In: Proc. of Computer Graphics International, CGI'2001, Hong-Kong (China). IEEE Computer Society Press, Los Alamitos, CA, (2001) 329-332.

[27] Iglesias, A., Echevarría, G., Gálvez, A.: Functional networks for B-spline surface reconstruction. Future Generation Computer Systems, 20(8) (2004) 1337-1353.

[28] Iglesias, A., Gálvez, A.: Curve fitting with RBS functional networks. In: Proc. of Int. Conference on Convergence Information Technology-ICCIT'2008 - Busan (Korea). IEEE Computer Society Press, 
Los Alamitos, CA, 1 (2008) 299-306.

[29] Kaur, A., Goyal, S.: A Bee Colony Optimization Algorithm for Fault Coverage Based Regression Test Suite Prioritization. International Journal of Advanced Science and Technology, 29 (2011) 17-30.

[30] Krishnasamy, V.: Genetic Algorithm for Solving Optimal Power Flow Problem with UPFC. International Journal of Software Engineering and Its Applications, 5(1), (2011) 39-50.

[31] G. K. Knopf, J. Kofman, Free-form surface reconstruction using Bernstein basis function networks. In: C. H. Dagli et al. (Eds.): Intelligent Engineering Systems Through Artificial Neural Networks. Vol. 9, ASME Press (1999) 797.

[32] Lee, T.C.M.: On algorithms for ordinary least squares regression spline fitting: a comparative study. J. Statist. Comput. Simul. 72(8) (2002) 647-663.

[33] Ma, W.Y., Kruth, J.P.: Parameterization of randomly measured points for least squares fitting of Bspline curves and surfaces. Computer Aided Design, 27(9) (1995) 663-675.

[34] Mahapatra, S., Jagadev, K., Naik, B.: Performance Evaluation of PSO Based Classifier for Classification of Multidimensional Data with Variation of PSO Parameters in Knowledge Discovery Database. International Journal of Advanced Science and Technology, 34 (2011) 27-44.

[35] Masrom, S., Abidin, S.Z.Z., Hashimah, P.N.: Towards Rapid Development of User Defined Metaheuristics Hybridization International Journal of Software Engineering and Its Applications, 5(2), (2011) 1-12.

[36] Patrikalakis, N.M., Maekawa, T.: Shape Interrogation for Computer Aided Design and Manufacturing. Springer Verlag, Heidelberg (2002).

[37] Piegl, L., Tiller, W.: The NURBS Book, Springer Verlag, Berlin Heidelberg (1997)

[38] Pottmann, H., Leopoldseder, S. Hofer, M., Steiner, T., Wang, W.: Industrial geometry: recent advances and applications in CAD. Computer-Aided Design 37 (2005) 751-766.

[39] Sawalha, R., Doush, I.A.: Face Recognition Using Harmony Search-Based Selected Features. International Journal of Hybrid Information Technology 5(2), (2012) 1-16.

[40] Sarfraz, M., Raza, S.A.: Capturing outline of fonts using genetic algorithms and splines. Proc. of Fifth International Conference on Information Visualization IV'2001, IEEE Computer Society Press (2001) 738-743.

[41] Singla, S., Kumar, D., Rai, M., Singla, P.: A Hybrid PSO Approach to Automate Test Data Generation for Data Flow Coverage with Dominance Concepts. International Journal of Advanced Science and Technology, 37 (2011) 15-26.

[42] Ssaed, A., Kadir, W., Hashim, S.Z.M.: Metaheuristic Search Approach Based on In-house/Outsourced Strategy to Solve Redundancy Allocation Problem in Component-Based Software Systems. International Journal of Software Engineering and Its Applications, 6(4), (2012) 143-154.

[43] Tyagi, Y., Puntambekar, T.A., Sexena, P., Tanwani, S.: A Hybrid Approach to Edge Detection using Ant Colony Optimization and Fuzzy Logic. International Journal of Hybrid Information Technology, 5(1), (2012) 37-46. 
[44] Ulker, E., Arslan, A.: Automatic knot adjustment using an artificial immune system for B-spline curve approximation. Information Sciences, 179 (2009) 1483-1494

[45] Varady, T., Martin, R.: Reverse Engineering. In: Farin, G., Hoschek, J., Kim, M. (eds.): Handbook of Computer Aided Geometric Design. Elsevier Science (2002).

[46] Varady, T., Martin, R.R., Cox, J.: Reverse engineering of geometric models - an introduction. Computer Aided Design, 29(4) (1997) 255-268.

[47] Worasucheep, C.: A Harmony Search with Adaptive Pitch Adjustment for Continuous Optimization. International Journal of Hybrid Information Technology, 4(4), (2011) 1-24.

[48] Yoshimoto F., Moriyama, M., Harada T.: Automatic knot adjustment by a genetic algorithm for data fitting with a spline. Proc. of Shape Modeling International'99, IEEE Computer Society Press (1999) 162-169.

[49] Zhao, X., Zhang, C., Yang, B., Li, P.: Adaptive knot adjustment using a GMM-based continuous optimization algorithm in B-spline curve approximation. Computer Aided Design, 43 (2011) 598-604.

\begin{abstract}
Authors
Akemi Gálvez is associate professor at the Department of Applied Mathematics

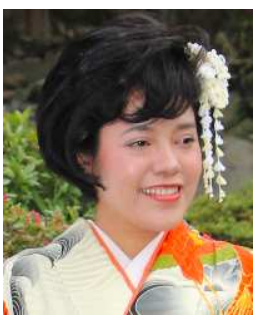
and Computational Sciences of the University of Cantabria (Spain). She holds a B.Eng. degree in Chemical Engineering in 1996. She also holds the M.Sc. and Ph.D. degrees in Computational Sciences at the University of Cantabria (Spain). She has published about 80 international papers on geometric modeling and processing, curve and surface reconstruction and soft computing, and has participated in several national and international projects on geometric modeling and processing and its applications to the manufacturing industry. She is also member of the editorial board of some international journals, and program committee member of several international conferences on computational science. Her main research fields are computer-aided geometric design, geometric processing, curve and surface reconstruction, artificial intelligence and soft computing.
\end{abstract}

Andrés Iglesias belongs to the Department of Applied Mathematics and Com-

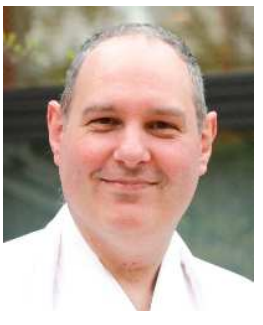
putational Sciences of the University of Cantabria (Spain), where he has been head of department for the period 2008-12 and post-graduate studies coordinator for seven years. He is also the founder and director of the Computer Graphics and Geometric Modeling research group at his institution. He holds a B.Sc. degree in Mathematics (1992) and a Ph.D. in Applied Mathematics (1995). He has published about 140 international papers and 13 books from Elsevier, SpringerVerlag, IEEE CS Press and Thomson Publishers. He has been the chairman and organizer of more than 40 international conferences and workshops in the fields of computational sciences, computer graphics, geometric modeling and symbolic computation. In addition, he has served as a steering committee member and program committee member in more than 150 international conferences, member of the editorial board of several international journals and reviewer of 140 conferences and more than 80 journal papers, mostly in ISI jour- 
nals. He has also been scientific expert evaluator of projects for the National Science Foundation (USA), the 7th. Framework Programme (European Union) and many other national and regional public research agencies in different countries. He has been visiting researcher in international institutions such as Wessex Institute of Technology-WIT (UK), International Center of Theoretical Physics-ICTP (Italy), and Izmir Institute of Technology (Turkey), and long-term visiting professor in the University of Tsukuba (Japan) and Toho University (Japan), as well as invited speaker in several national and international conferences. He is an international committee member of the IFIP Technical Committee 5- Information Technology Applications, Workgroup 5.10 - Computer Graphics and Virtual Worlds. His research interests are in the fields of computer graphics, geometric modeling, CAD/CAM, computer animation and videogames, artificial intelligence, symbolic computation, and soft computing.

Luis Cabellos graduated as a Computer Science Engineer at the University of

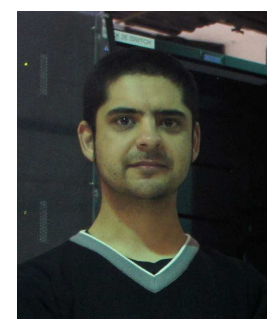
Oviedo (Spain) in 2003. He also holds a M.Sc. in Computer Science at the University of Cantabria (Spain) in 2010. Currently, he works at the Instituto de Física de Cantabria (IFCA), particularly in the Advanced Computing \& eScience Research Line at IFCA, where he is the User Support and System Administrator of the Supercomputing node Altamira, an integral part of the Spanish Supercomputing Network (RES). He has published some international papers in the fields of geometric modeling and high-performance computing. His research interests include supercomputing, high-performance computing, distributed and parallel computing, computer graphics and geometric modeling. 
International Journal of Software Engineering and Its Applications Vol.7, No.5 (2013) 